Volume: 2, Issue: 3, September 2017, Pages: 201-206, DOI: http://dx.doi.org/10.19082/ah201

\title{
AN EPIDEMIOLOGICAL STUDY OF SUPERFICIAL FUNGAL INFECTIONS IN PATIENTS REFERRED TO YAZD CENTRAL LABORATORY, IRAN
}

\author{
Mohammad Ebrahimzade Ardekani ${ }^{1}$, Naser Ghaderi ${ }^{2}$, Mahmood Vakili $^{3}$, Sepehr Nabavinejad ${ }^{4}$
}

1: M.D., Associate professor, Department of Dermatology, Shahid Sadoughi University of Medical Science, Yazd, Iran

2: M.D., Department of Dermatology, Shahid Sadoughi University of Medical Science, Yazd, Iran

3: M.D., MPH., Department of Community Medicine, School of Medicine, Shahid Sadoughi University of Medical Sciences, Yazd, Iran

4: M.D., Department of Radiology, School of Medicine, Shahid Sadoughi University of Medical Sciences, Yazd, Iran

TYPE OF ARTICLE: ORIGINAL ARTICLE

\begin{abstract}
Introduction: Superficial and cutaneous infections have high prevalence all over the world. It is necessary to be informed of disease prevalence in each zone and be able to identify its causes and dominant species to obtain proper diagnosis, early treatment, planning control, and prophylaxis. The main objective of the present paper is to determine the frequency of fungal infections and their agents and identify different kinds of dermatophytes and their distribution patterns in patients referred to Yazd Central Laboratory, Iran.

Methods: In this retrospective descriptive study, patients suspected of having superficial fungal disease were surveyed from March 2011 to March 2013. Samples were taken from lesions, and diagnosis was made by means of fungal culture or direct microscope. In the case of observation of yeast, hyphae, mycelium, arthroconidia, spore, pseudohyphae, and budding cells in the samples, it was considered through positive smear result. The main values of this study were data analyzed by descriptive statistics in SPSS version 17 software; also, a chi-square test was used.

Results: 2203 patients were studied: 685 patients (31.1\% of all patients) suffered from superficial and cutaneous fungal infection; 498 patients (72.7\%) suffered from dermatophytosis, the most prevalent infection, and respectively Cutaneous candidiasis (14.3\%), erythrasma (10.3\%), Tinea versicolor (2\%), and nail aspergillus $(0.7 \%)$ were next. In 125 cases, the upper limb was infected $(25.1 \%)$.

Conclusion: According to this study, dermatophytosis is the most prevalent and important fungal infection; thus, we should focus on educating and improving the hygiene of the societies in question to prevent and reduce the prevalence of these diseases.
\end{abstract}

KEYWORDS: Epidemiology, Dermatophytosis, Fungal infection, Yazd

\section{INTRODUCTION}

In terms of colony shape, fungi can be divided into two categories: yeasts and molds. However, some fungi are able to produce both of these forms (dimorphic) (1). As far as the anatomic location of the primary target of fungi is concerned, fungal infections can be classified into four groups, i.e., superficial, cutaneous, subcutaneous, and systemic or visceral $(1,2)$. Superficial and cutaneous fungal infections are actually fungal skin diseases that are caused by fungi such as dermatophytes and some opportunistic ones such as malassezia, candida, and aspergillus. Prevalence and characteristics of these disease vary with environmental conditions, lifestyle, public immigration conditions, etc. (2). Dermatophytosis or ringworm is one of the most important and prevalent cutaneous fungal diseases and involves infections that are caused exclusively by a group of keratinophilic fungi named dermatophytes (3). Pathological changes caused by these infections are the result of the host's immune system response to fungi agents and their metabolic products (4). So far, more than 40 species of dermatophyte have been identified that belong to three genera of microsporum, trichophyton, and epidermophyton (5). Only 11 of the 40 species of dermatophyte species are common causes of ringworm in humans, which originates from human, animal, or soil (3).

\section{Correspondence:}

Dr. Naser Ghaderi, Department of Dermatology, Shahid Sadoughi University of Medical Science, Yazd, Iran. Tel: +98.3538229007, Email: dr.naserghadery@yahoo.com

Received: October 29, 2016, Accepted: June 7, 2017, Published: September 2017

iThenticate screening: October 30, 2016, English editing: June 22, 2017, Quality control: July 8, 2017

(C) 2017 The Authors. This is an open access article under the terms of the Creative Commons Attribution-NonCommercialNoDerivs License, which permits use and distribution in any medium, provided the original work is properly cited, the use is non-commercial and no modifications or adaptations are made. 
Dermatophytosis has multiple clinical forms that can be classified into the following types according to the site: body (T.corporis), scalp and hair (T.capitis), facial hair (T.barbae), face (T.faciei), feet (T.pedis), hands and palm area (T.Mannum), the groin area (T.cruris), and fingernails and toenails (T.unguinum).

It is estimated that $10 \%-20 \%$ of people experience these infections at least once in their life. Distribution of these infections is different in various countries, and they are more prevalent in developing countries than in industrial ones (5). Prevalence of fungal infections in different areas depends on the health, cultural, and economic conditions of each area (4). For correct diagnosis and timely treatment and also planning for control and prevention of these infections, it is necessary to have enough information and knowledge on the prevalence of these diseases and the dominant agents and species causing them in each region. The reports from various studies carried out in different cities and countries suggest that geographical distribution of superficial and cutaneous fungal infections and particularly dermatophytosis is different not only from region to region but also from year to year (1-5). Hence, the main objective of the present paper is to determine frequency of fungal infections and their agents and identify different kinds of dermatophytes and their distribution patterns in patients referred to Yazd Central Laboratory, Iran.

\section{MATERIALS AND METHODS}

\subsection{Research Design and Setting}

A retrospective, descriptive study was carried out in the period January 2011-2013 on 2203 patients with superficial skin lesions suspected to fungal infection referred to the Mycology Department of the Yazd Central Laboratory, Iran.

\subsection{Participants}

Observation of clinical symptoms along with at least one positive test result was determined to be considered "fungal infection diagnosis". All patients who visited the Mycology Department of Yazd Central Laboratory, Iran, for fungal infection diagnosis were included with census sampling. Suspicious lesions in the right place were sampled in the case of not taking a bath at least three days prior to admission and not taking antifungal drug. Otherwise, the patients were provided with necessary recommendation, and the sampling was repeated in the next visit. Patients with an uncomplete medical history were exluded.

\subsection{Data Collection}

In terms of scalp and hair lesions, first, the hair was cut short with a pair of scissors; after cleaning the site, the infected hair was removed with a pair of forceps. About the lesions without hair, the place was cleaned with sterile gauze soaked in alcohol, and then the skin on the side of lesion that is usually more active was shaved with a sterile scalpel. Samples were collected on a clean and sterile slide or in a plate. At the end, skin and nail samples with $10 \%$ potassium hydroxide $(\mathrm{KOH})$ and hair samples with lactophenol solution were prepared and then analyzed using an optical microscope after half an hour or an hour. In corrugated body areas, in addition to the KOH smear, extra sampling and staining with methylene blue were done for diagnosis of erythrasma. The mediums were kept at laboratory temperature, and macroscopic and microscopic evaluation of fungal growth was conducted on them by preparing some slides. Type of pathogenic fungi was identified based on the appearance of colony or through preparation of a wet sample of the colony with cutton-blue lactophenol solution and, if necessary, by on-slide culture method. In the case of no fungal agent growth after four weeks, the culture result was considered negative. In the case of observation of yeast, hyphae, mycelium, arthroconidia, spore, pseudohyphae, and budding cells in the samples, it was considered a positive smear result. Existence of hyphae, mycelium, or arthroconidia is indicative of dermatophytes, and existence of yeast, pseudohyphae, and budding cells is a sign of infections such as candida and malassezia. Sabourud dextrose agar containing chloramphenicol (SC) and Sabourud dextrose agar containing chloramphenicol and cyclohexamide (SCC) were used as mediums for culture of samples.

\subsection{Research Ethics}

This study was approved by the ethical committee of Shahid Sadoughi University of Medical Sciences, Yazd. The study was submitted to an ethical committee (submission number 785362). There were no names on the patients' data, which were collected and all data secretly kept.

\subsection{Statistical Analysis}

Data analyzed by descriptive statistics in SPSS Statistics for Windows, Version 17.0 (Released 2008. Chicago: SPSS Inc). The results were presented in frequency tables; also, Chi-squre test was used. 


\section{RESULTS}

Among 2203 patients, 1122 (50.9\%) were male and 1081 (49.1\%) were female. According to the results, 1714 patients $(77.8 \%)$ had only KOH smear and 489 patients $(22.1 \%)$ had both KOH smear and fungus culture. Samples were collected from scalp (12.1\%), face (7.4\%), neck (4.2\%), upper extremities (20.1\%), armpit (18.1\%), back $(2.4 \%)$, chest and abdomen together (11.0\%), genitals (1.2\%); groin (6.5\%), lower extremities, and toenails and fingernails together $(17.0 \%)$.

Resutls showed that $685(31.1 \%)$ had one of the types of superficial and cutaneous fungal infections. Dermatophytosis with 498 cases $(72.7 \%)$ and nail aspergillus with five cases $(0.72 \%)$ were the most and the least prevalent infections among the subjects. Among 685 subjects with superficial fungal infection, 349 (50.95\%) were male and $336(49.05 \%)$ were female. A significant relationship was found between gender and Tinea versicolor $(p=0.0001)$, cutaneous candidiasis $(p=0.02)$, and erythrasma $(p=0.03)$ (Table 1$)$.

Table 1: Distribution of superficial and cutaneous fungal infections patients according to the gender

\begin{tabular}{|l|l|l|l|}
\hline Type of infection, $\mathrm{n}(\%)$ & Male $(\mathrm{n}=349)$ & Female $(\mathrm{n}=336)$ & $\mathrm{p}$-value \\
\hline Dermatophytosis & $271(54.42)$ & $227(45.58)$ & 0.45 \\
\hline Cutaneous candidiasis & $28(28.6)$ & $70(71.4)$ & 0.02 \\
\hline Erythrasma & $44(62.86)$ & $26(37.14$ & 0.03 \\
\hline Tinea versicolor & $4(28.57)$ & $10(71.43)$ & 0.001 \\
\hline Nail aspergillus & $2(40)$ & $3(60)$ & 0.64 \\
\hline
\end{tabular}

Dermatophytosis was the main fungal infectious of the scalp, which is significantly more than the others $(p=0.001)$ (Table 2). The maximum and minimum cases of superficial and cutaneous fungal infections, respectively, belong to nails with 139 cases $(20.3 \%)$ and genitals with nine cases (1.3\%) (Table 2). Among various types of ringworm (dermatophytosis), upper extremities with 125 cases $(25.1 \%)$ and genitals with one case $(0.2 \%)$ showed the highest and the lowest frequencies. The highest frequency of different types of cutaneous candidiasis was observed in nails of subjects with 74 cases $(75.51 \%)$. In addition, the highest frequencies of erythrasma and Tinea versicolor were observed in the groin with 40 cases (57.15\%) and trunk (chest, back, and abdomen) with seven cases, respectively. Only five cases of aspergillus were found among saprophyte nail infections.

Table 2: Distribution of superficial and cutaneous fungal infections based on the location of the lesion

\begin{tabular}{|l|l|l|l|l|l|l|}
\hline $\begin{array}{l}\text { Position of infection, } \\
\mathrm{n}(\%)\end{array}$ & $\begin{array}{l}\text { Dermatophytosis } \\
(\mathrm{n}=498)\end{array}$ & $\begin{array}{l}\text { Cutaneous } \\
\text { candidiasis }(\mathrm{n}=98)\end{array}$ & $\begin{array}{l}\text { Erythrasma } \\
(\mathrm{n}=70)\end{array}$ & $\begin{array}{l}\text { Tinea versicolor } \\
(\mathrm{n}=14)\end{array}$ & $\begin{array}{l}\text { Nail aspergillus } \\
(\mathrm{n}=5)\end{array}$ & $\begin{array}{l}\mathrm{p}- \\
\text { value }\end{array}$ \\
\hline Scalp & $72(14.45)$ & 0 & 0 & 0 & 0 & 0.001 \\
\hline Face & $34(6.8)$ & $1(1.02)$ & 0 & $2(14.3)$ & 0 & 0.001 \\
\hline Neck & $19(3.8)$ & $1(1.02)$ & 0 & $1(7.15)$ & 0 & 0.001 \\
\hline Upper extremities & $125(25.1)$ & $2(2.05)$ & 0 & $3(21.4)$ & 0 & 0.001 \\
\hline Axilary & $2(0.04)$ & $4(4.8)$ & $12(17.15$ & $1(7.15)$ & 0 & 0.67 \\
\hline Back, chest, abdomen & $47(9.45)$ & $3(3.06)$ & $5(7.15)$ & $7(50)$ & 0 & 0.035 \\
\hline Groin & $46(9.25)$ & $10(10.2)$ & $40(57.15)$ & 0 & 0 & 0.58 \\
\hline Genitalia & $1(0.2)$ & $3(3.06)$ & $5(7.15)$ & 0 & 0 & 0.89 \\
\hline Lower extremities & $92(18.5)$ & 0 & $8(11.4)$ & 0 & 0 & 0.002 \\
\hline Nails & $60(12.05)$ & $74(75.51)$ & 0 & 0 & $5(100)$ & 0.87 \\
\hline
\end{tabular}

\section{DISCUSSION}

The high prevalence of superficial and cutaneous fungal infections around the world shows the importance of these diseases. In the present study, prevalence of superficial and cutaneous fungal infections was obtained to be $31.1 \%$ among 2203 patients referred to the Yazd Central Laboratory during the three studied years. In Iran, many studies and surveys have been conducted on the statistics and prevalence of superficial fungal infections and especially dermatophytosis in various provinces. In a similar study conducted by Nasrollahi et al. (2009) in Tehran, the prevalence of superficial fungal infections was determined to be 41.29\% (1). In addition, Jafari (2003) in Yazd (1), Azizi (2001) in Yazd (2), and Moshir (2001) in Tehran (3) reported that the prevalence of superficial fungal infections is $40 \%, 25.4 \%$, and $50.6 \%$, respectively. These data are nearly similar due to the close proximity of the population. In the present study, prevalence in males $(50.95 \%)$ was a little more than in the females, which is somewhat consistent with the findings of Jafari (4) and Azizi (2) in Yazd but inconsistent with the findings of 
Nasrollahi (1) and Moshir (3), who reported that the prevalence of superficial fungal infections in females in more than males. We cannot focus on this result due to changes in population and sanctions in the different parts of the city. In the present study, ringworm or dermatophytosis $(72.7 \%)$ was the most frequent fungal infection among other ones. Cutaneous candidiasis (14.3\%), erythrasma (10.3\%), Tinea versicolor $(2 \%)$, nail saprophyte infections like aspergillus (0.7\%), respectively, ranked after dermatophytosis. In the study, Nasrollahi et al. (2009) in Tehran (1), dermatophytosis $(56.31 \%)$ was the most prevalent superficial fungal infection, and Tinea versicolor (15.68\%), erythrasma (12.76\%), Cutaneous candidiasis $(10.7 \%)$, and nail saprophyte infections $(4.96 \%)$ ranked next, respectively.

In the study conducted by Jafari in Yazd (4), dermatophytosis (43.9\%) was found to be the most prevalent superficial fungal infection, and Tinea versicolor (33.6\%), erythrasma (10.4\%), cutaneous candidiasis (6.3\%), and saprophytes (1.1\%), respectively, ranked after dermatophytosis. In the study carried out by Moshir in Tehran (5), dermatophytosis (51.4\%), erythrasma (20.1\%), cutaneous candidiasis $(18.6 \%)$, and Tinea versicolor $(8.3 \%)$ were the most prevalent superficial fungal infections, respectively. Azizi (2) also reported that dermatophytosis is the most frequent superficial fungal infection among the studied population in Yazd. In a study carried out in Turkey, among 8200 patients with suspected fungal infections, 5722 of them were afflicted with these infections. Dermatophytosis $(74 \%)$, candidiasis $(21 \%)$, and Tinea versicolor $(3 \%)$, respectively, were the most prevalent fungal infections in the studied population (6). In a study conducted in Japan in 2002, dermatophytosis was reported to be the most prevalent superficial fungal infection, and Cutaneous candidiasis and Tinea versicolor ranked second and third, respectively (9).

According to the results of the present study and comparing them with findings of other studies, it can be concluded that dermatophytosis is the most prevalent superficial and cutaneous fungal infection. According to the results of this study, among the 498 patients with dermatophytosis, upper extremities with 125 cases $(25.1 \%)$ were the most common type of problem observed in these patients. Then, lower extremities with 92 cases $(18.5 \%)$ ranked second among dermatophyte infections. Ringworm in scale and hair with 72 cases $(14.45 \%)$, nails ringworm with 60 cases (12.05\%), trunk ringworm with 47 cases $(9.45 \%)$, and groin ringworm with 46 cases $(9.25 \%)$ ranked next among the dermatophyte infections, respectively. In the study conducted by Azizi in Yazd (2), ringworm in scale and hair, trunk, hands, and feet showed the highest frequency in the studied region. Jafari (4) reported that the most prevalent dermatophyte infection in Yazd is related to upper extremities with 192 cases, which is consistent with the findings of the present study. Head and face, groin, and upper extremities ranked next, respectively. In the studied conducted by Mousavi in Kerman (5), hand ringworm was found to be the most prevalent problem observed in the studied patients. In the study carried out by Mollaei in Zahedan (10), scalp and hair ringworm (61\%) was reported to be the most prevalent clinical type of dermatophytosis. In the study of Nasrollahi (2), among 1279 cases of dermatophytosis, groin ringworm (35.8\%) was the most frequent problem, and then nail ringworm (20.7\%), hand and feet ringworm (11.72\%), and scalp and hair ringworm (7.8\%) ranked next, respectively. In a study carried out by Edalatkhah et al. (11), trunk ringworm was found to be the most frequent form of dermatophytosis.

In a study conducted in 2008 in Saudi Arabia (7), nail ringworm (40.3\%) was reported to be the most prevalent clinical type of dermatophytosis, and scalp and hair ringworm, feet ringworm, and trunk ringworm ranked next, respectively. In two studies conducted in Japan in 2002 (8) and Mexico in 2006 (9), feet ringworm was found to be the most frequent type of ringworm. Among the 489 cases of requested fungus culture in the present study, the result of 100 tests $(20.5 \%)$ was positive. Dermatophyte fungi with 62 cases of culture were the most frequent fungi isolated, among which Trichophyton verrucosum, a zoophilic fungus, with 22 cases and Trichophyton mentagrophytes with 19 cases were the most prevalent species. In the studies conducted by Jafari and Azizi in Yazd $(1,2,4)$, the most prevalent fungal species were Trichophyton verrucosum and Trichophyton violaceum, respectively. The result of Jafari in this regard is consistent with the finding of the present study, while the species found by Azizi as the most prevalent fungal species was the rarest species of dermatophyte in the present study. In a study carried out by Edalatkhah et al. (10), Trichophyton verrucosum was reported as the most prevalent fungal species. Trichophyton mentagrophytes ranked second in the present study, while Nasrollahi (2), Mousavi (5), Moallaei (10), and Baghestani (11) introduced it as the most prevalent fungal species in their studies. In the study by Aghamiran in Qazvin (12), Epidermophyton floccosum was reported to be the most prevalent agent of dermatophytosis, while it ranked third in the present study. In the studies conducted in Japan (8), Mexico (9), and Turkey (6), Trichophyton rubrum has been reported to be the most common fungal agent in dermatophytosis, while there was no record of this species in the present study. 
The differences in frequency of clinical types and various species involved in dermatophytosis observed in various studies could be attributed to individual and community health status, lifestyles, climatic conditions, contact with animals, immigration, and other factors (12-16). Cutaneous candidiasis is one of the most common fungal infections. In the present study, Cutaneous candidiasis with 98 cases ranked second after dermatophytosis among the fungal infections, while this disease ranked fourth in the studies conducted by Nasrollahi (1), Azizi (2), and Jafari (4). Cutaneous candidiasis was more prevalent in women than men, and the frequent clinical form of this disease was found to be onychomycosis. This is consistent with the findings of other studies. Erythrasma is a superficial bacterial infection that mostly occurs in the corrugated areas of the body. In the present study, erythrasma ranked third with 70 positive cases. It has also ranked the same in the studies conducted by Nasrollahi (2), Azizi (2), and Jafari (4). Prevalence of erythrasma was higher in men than in women; the groin showed the highest frequency of this infection. This is consistent with the findings of other studies. Among the 685 fungal infections identified in this study, only subjects were afflicted with Tinea versicolor, so it ranked fourth. In studies conducted by Nasrollahi (1), Jafari (4), and Azizi (2), this infection was reported to be the second most prevalent infection with 356, 504, and 15 cases, respectively. Tinea versicolor is mostly diagnosed clinically and is referred to the laboratory for confirmation in few cases. This would be one of the reasons for low frequency of cases with Tinea versicolor in the present study. In this study, we have a lot of missing data because of lack of records in the data base, and we can suggest undertaking more studies in this field with a bigger data set and also with better databases. For the next studies, it will be ideal to have more cultures and more patients.

\section{CONCLUSIONS}

According to the results of this research, it can be concluded that cutaneous and superficial fungal infections are still of high prevalence, and dermatophytosis is the most common and important fungal infection. In this study, zoophilic fungi had the highest frequency. Therefore, direct contact with infected animals and lack of observance of personal hygiene are important factors involved in afflictions with this type of fungal infections. To prevent these infections and reduce the prevalence of these diseases, special attention should be paid to training of the individuals on fungal diseases and their transmission ways, observance of social and personal hygiene, and identification and treatment of infected animals.

\section{ACKNOWLEDGMENTS:}

The authors would like to thank Dr. Akhavan and Dr. Hekmati, the former and current directors of Yazd Central Laboratory, Mr. Khajeh Afzali, the Supervisor Yazd Central Laboratory, and Ms. Mandegari, the head of Mycology Department of Yazd Central Laboratory for their sincere assistances.

\section{CONFLICT OF INTEREST:}

The authors declare that they have no conflict of interests.

\section{AUTHORS' CONTRIBUTIONS:}

All authors contributed to this project and article equally. All authors read and approved the final manuscript.

\section{REFERENCES:}

1) Khanna N, Stuehler C, Lunemann A, Wojtowicz A, Bochud PY, Leibundgut-Landmann S. Host response to fungal infections - how immunology and host genetics could help to identify and treat patients at risk. Swiss medical weekly. 2016; 146: w14350. doi: 10.4414/smw.2016.14350.

2) Mariette C, Tavernier E, Hocquet D, Huynh A, Isnard F, Legrand F, et al. Epidemiology of invasive fungal infections during induction therapy in adults with acute lymphoblastic leukemia: a GRAALL-2005 study. Leukemia \& lymphoma. 2016: 1-8 .

3) Obradovic-Tomasev M, Jovanovic M, Vuckovic N, Popovic A. Fungal infections in corn picker hand injury. Srpski arhiv za celokupno lekarstvo. 2016; 144(1-2): 52-5. doi: 10.2298/SARH1602052O. PMid: 27276858.

4) Vallabhaneni S, Chiller TM. Fungal Infections and New Biologic Therapies. Current rheumatology reports. 2016; 18(5): 29. doi: 10.1007/s11926-016-0572-1. PMid: 27032792.

5) Visentin A, Gurrieri C, Imbergamo S, Lessi F, Di Maggio SA, Frezzato F, et al. Epidemiology and risk factors of invasive fungal infections in a large cohort of patients with chronic lymphocytic leukemia. Hematological oncology. 2016. doi: 10.1002/hon.2343. PMid: 27641225.

6) Nasrollahi Omran A, Hashemi SJ, Hashemi F. Epidemiology of superficial and cutaneous mycosis in 5500 suspected patients in Tehran. Tehran University Medical Journal TUMS Publications. 2010; 68(1): 45-53. 
7) Azizi M, Jivad N. Causal agents of the prevalent cutaneous fungal diseases in Yazd province, 1998. J Shahrekord Univ Med Sci 2001, 3(2): 73-78 .

8) Tabatabai A, Poureslami M, Shamshiri A, Moshir M. Prevalance Rate Of Different Types Of Yeast Infection in Patients Visiting the Dermatology Clinic of Hazrat Rasul-E Akram Hospital. Razi Journal of Medical Sciences. 2002; 8(26): 466-70.

9) Jafari-nodoushan AA, Kazemi A, Mirzaii F, Dehghani M. Fluconazole susceptibility profile of Candida isolates recovered from patients specimens admitted to Yazd Central Laboratory. Iranian Journal of Pharmaceutical Research. 2010: 69-75.

10) Koksal F, Er E, Samasti M. Causative agents of superficial mycoses in Istanbul, Turkey: retrospective study. Mycopathologia. 2009; 168(3): 117-23. doi: 10.1007/s11046-009-9210-z. PMid: 19544086.

11) Abanmi A, Bakheshwain S, El Khizzi N, Zouman AR, Hantirah S, Al Harthi F, et al. Characteristics of superficial fungal infections in the Riyadh region of Saudi Arabia. International journal of dermatology. 2008; 47(3): 229-35. doi: 10.1111/j.1365-4632.2008.03563.x. PMid: 18289321.

12) Nishimoto K. [An epidemiological survey of dermatomycoses in Japan, 2002]. Nihon Ishinkin Gakkai zasshi= Japanese journal of medical mycology. 2005; 47(2): 103-11.

13) Welsh O, Welsh E, Ocampo-Candiani J, Gomez M, Vera-Cabrera L. Dermatophytoses in monterrey, méxico. Mycoses. 2006; 49(2): 119-23. doi: 10.1111/j.1439-0507.2006.01199.x. PMid: 16466445.

14) Edalatkhah H, Golforoshan F, Azimi H, Mohammadi P, Razi A. Prevalence of various species of dermatophytes in patients referring to the dermatology clinic of Tabriz Haft-e-Tir Hospital. Journal of Ardabil University of Medical Sciences. 2006; 6(1): 47-52.

15) Mahboobi A, Baghestani S, Hamedi Y, Heidari M, Vahdani M. Epidemiology of dermatophytosis in Bandar Abbas, Iran 2003-2004. Bimonthly Journal of Hormozgan University of Medical Sciences. 2006; 9(4): 227-34.

16) Aghamirian MR, Keshavarz D, Jahani Hashemi H. Clinical evaluation of dermatophytosis in patients referred to dermatologic department of Bu-Ali Sina Hospital in Qazvin in Iran 2004-2005. ISMJ. 2007; 9(2): $175-81$. 